\title{
Optimization and analysis of some variants through Vogel's approximation method (VAM)
}

\author{
Shweta Singh ${ }^{1}$ G.C. Dubey ${ }^{2,}$ Rajesh Shrivastava ${ }^{3}$ \\ ${ }^{1}$ Radharaman Institute of Technology and Science, Bhopal India \\ ${ }^{2}$ Govt. M.G.M. College, Itarsi (M.P.), India \\ ${ }^{3}$ Govt. Science and Commerce College, Benazir, Bhopal, India
}

\begin{abstract}
VAM is one of the most efficient solution to the transportation problems. In VAM Variants are proposed using total opportunity cost and allocation cost. Through the survey of computational experiments the results are evaluated through VAM and improved version of VAM Performance if improved version of VAM over VAM is also discussed and analysis through iterations. Total opportunity cost in transportation has been studied through critical problem in industries, military etc. In Analysis we found that the quality of solution in basic version of VAM coupled with total opportunity cost yields a very efficient initial solution. In survey experiment on an average about $20 \%$ of the time, the VAMTOC yields the optimal solution and about $80 \%$ of the time it yields to a solution nearest to the optimal Value. A comparative study is also shown through various charts.
\end{abstract}

Keywords_Initial basic Feasible solution (IBFS), Transportation problem, Vogel's Approximation method, Total opportunity cost (TOC).

\section{INTRODUCTION}

Transportation models deal with problems concerning as to at happens to the effectiveness function. When we associated each of origins (Sources) with each of a possibly different umber of destinations (jobs). The total movement from each origin and the total movement to each destination is given and it is desired to find how the associations be made subject to the limitations on totals.

The transportation problem deals with the transportation of a product manufactured at different plants or factories (supply of origin) to a number of different ware houses. (Demand, destinations). The objective is to satisfy the destination requirement within the plant's capacity constraints at the minimum transportation cost.

The transportation problem constitutes on important part of logistics management and also a special kind of the network optimization problem. Transportation models play an important role in logistics and supply chains. The easiest way to recognize a transportation problem is to consider a typical situation as shown in the following figure. Assume that a manufacturer has three factories $F_{1}, F_{2}, F_{3}$ producing the same product, from these factories, the product is transported to three warehouses $\mathrm{W}_{1}, \mathrm{~W}_{2}$ and $\mathrm{W}_{3}$, each factory has limited supply and each warehouse has specific demand. Each factory can transport to each warehouse but the transportation cost vary for different combinations the problem is to determine the quantity each factory. Should transport to each ware house in order to minimize total transportation Cost

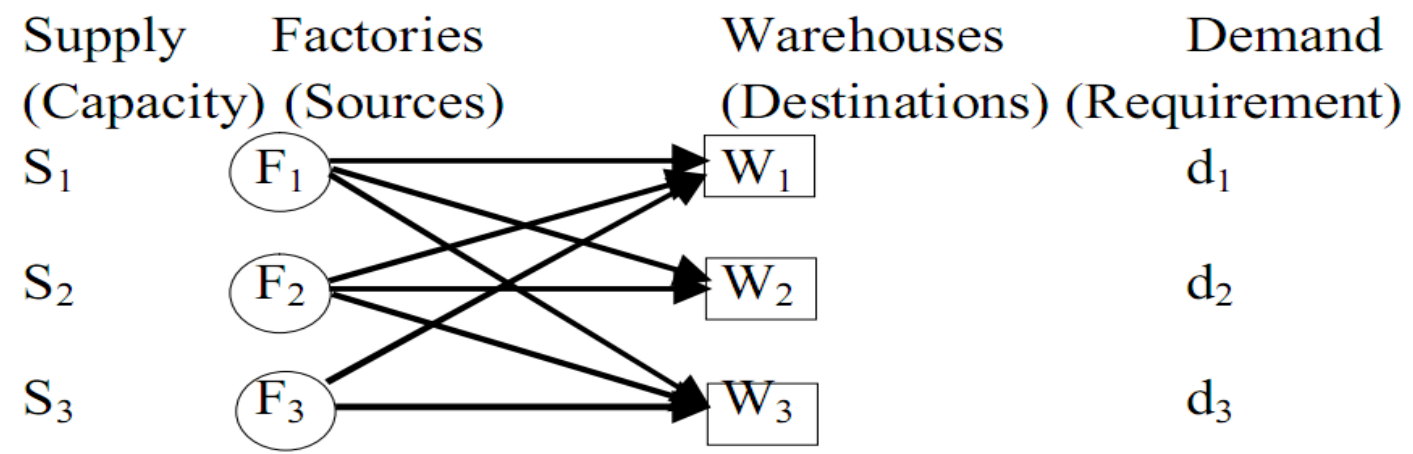

Fig.1 Example of a Transportation problem 


\subsection{Structure and formulation of the Transportation Problem}

An important feature of the standard transportation problem is that it can be expressed in the form of a table, which displays of all values of data coefficients $\left(s_{i}, d_{j}, c_{i j}\right)$ associated with the problem

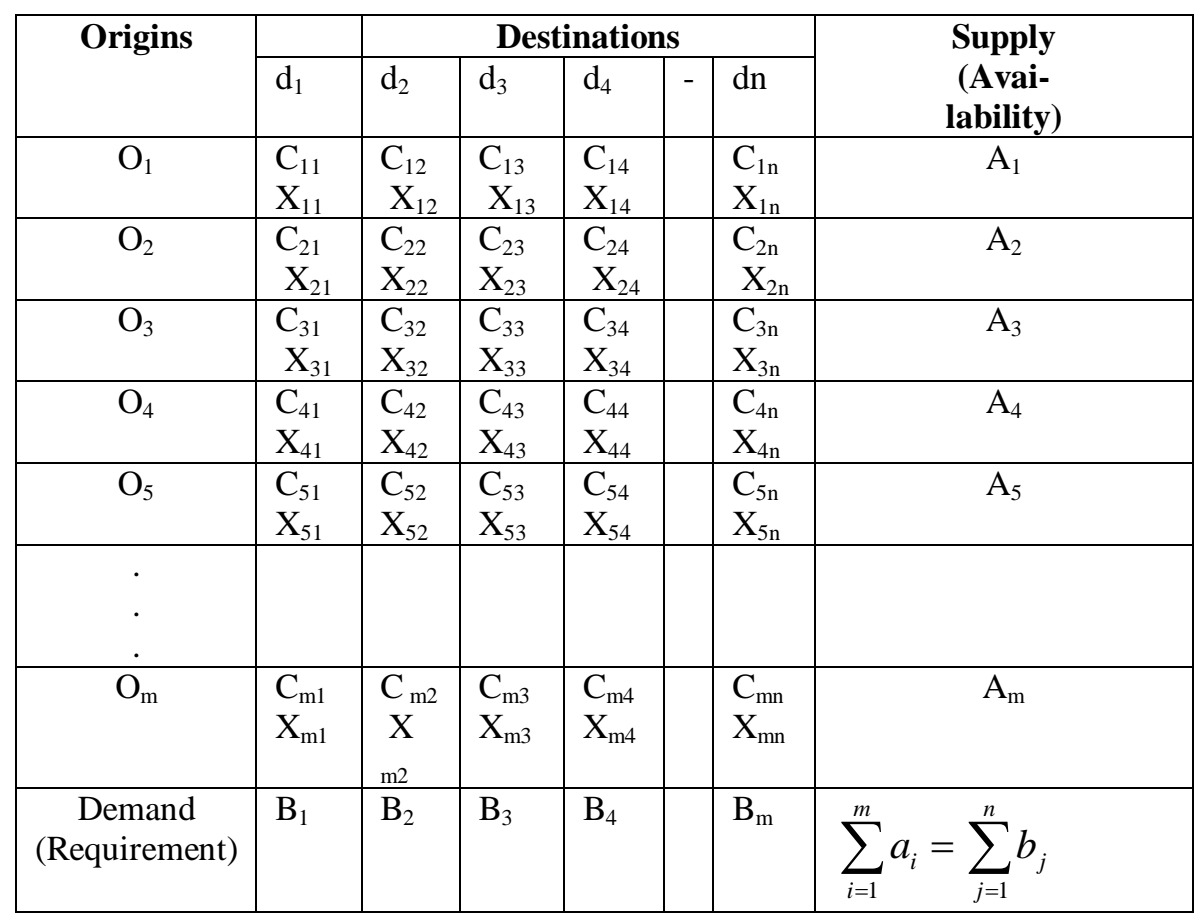

Fig.2 General Transportation Table Structure

Formulation of the linear programming model

Minimize Z $=\sum_{i=1}^{m} \sum_{j=1}^{n} x_{i j} c_{i j}$

Subject

to

$$
\sum_{i=1}^{m} x_{i j}=a_{i} \quad \mathrm{i}=1,2,3,-\mathrm{m}
$$

(1) (Capacity constraint)

$$
\sum_{j=1}^{n} x_{i j}=b_{i} \quad \mathrm{j}=1,2,3,-\mathrm{n}
$$

(2) (Requirement Constraint)

$$
x_{i j}>0
$$

Where

The two sets of constraints will be consistent ie the system will be balance if

$$
\sum_{i=1}^{m} a_{j}=\sum_{j=1}^{n} b_{j}
$$

Thus eq (3) is necessary as well as a sufficient conditions for a transportation problem to have a feasible solution. Problems that satisfy this condition are called balanced. Transportation problem (BTP) 
Techniques have been developed for solving BTP or STP (stand are transportation problem). The reader may refer to Wagner [1] and Taha [2] for detailed coverage of T.P.

The following terms are to be defined with reference to the transportation problem

[A] Feasible Solution (F.S.)

A non-negative allocation xij zo which satisfy the row and column restrictions is known as F.S.

[B] Basic Feasible solution (B.F.S.)

A feasible solution to $\mathrm{m}$-origin and $\mathrm{n}$-destination problem is said to be B.F.S. if the number of positive allocation are $(m+n-1)$

If the number of allocation in a basic feasible solution are less than $(m+n-1)$ it is called degenerate B.F.S. (D.B.F.S.) otherwise non-degenerate

[C] Optimal Solution(O.S.):-

A feasible solution (not necessarily basic) is said to be optimal if it minimizes the total transportation cost

The Basic steps to solve transportation problem are:

Step

1. To determine the IBFS (Initial basic feasible solution)

2. To determine the optimal solution using IBFS.

As IS (Initial solution) is a first attempt to match supply and demand along advantageous distribution routes. Also there are several methods available to obtain on initial solution (IS). Well-known heuristics methods are North West Corner [3], Lowest cost entry method VAM method [4], Shimshak et. Als version of VAM [5], Goyal's version of VAM [6], Ramkrishnan's version of VAM [7] etc. Kirca and Satir [8] developed a heuristics to obtain efficient IBFS is called TOM. Bala Krishnan [9] Proposed a modified version of VAM for unbalanced Transportation problem. Sharma and Prasad [10] proposed heuristics gives significantly better solutions than the well know VAM. Mathirajan and Meenakshi [11] were extended. TOM using the VAM procedure.

\subsection{Variant's of Vogel's approximation method and the allocation:}

VAM is a heuristics and usually provides a better starting solution than other methods. Application of VAM to a give problem does not guarantee that an optimal solution will result. However, a very good solution is invariably obtained with comparatively little effort [12].

This method also takes cost into account in allocation five steps are involved in applying this heuristics

Step:1. Determine the difference between the lowest two cells in all rows and columns, including dummies.

Step:2. Identify the row or column with the largest difference ties may be broken arbitrarily.

Step:3. Allocate as much as possible to the lowest-cost cell in the row or column with the highest differences. If two or more differences are equal, allocate as much as possible to the lowest-cost cell in these rows or columns.

Step:4. Stop the process if all row and go to next step column requirement are met, if not

Step:5. Recalculate the differences between the two lowest cells remaining in all rows and columns. Any row and column with zero supply demand should not be used in calculating further differences than go to step-2

The VAM usually produces an optimal or near-optimal starting solution. One study found that VAM yields an optimum solution in $80 \%$ of the sample problems tested.

\section{Example-1}

To find the Initial basic feasible solution (IBFS) by VAM method

Table- 1

\begin{tabular}{|l|l|l|l|l|l|l|}
\hline & $\mathrm{D}_{1}$ & $\mathrm{D}_{2}$ & $\mathrm{D}_{3}$ & $\mathrm{D}_{4}$ & $\mathrm{D}_{5}$ & Supply source \\
\hline $\mathrm{O}_{1}$ & 68 & 35 & 4 & 74 & 15 & 18 \\
\hline $\mathrm{O}_{2}$ & 57 & 88 & 91 & 3 & 8 & 17 \\
\hline $\mathrm{O}_{3}$ & 91 & 60 & 75 & 45 & 60 & 19 \\
\hline $\mathrm{O}_{4}$ & 52 & 53 & 24 & 7 & 82 & 13 \\
\hline $\mathrm{O}_{5}$ & 51 & 18 & 82 & 13 & 7 & 15 \\
\hline Required & 16 & 18 & 20 & 14 & 14 & $82 / 82$ \\
\hline
\end{tabular}


Table-2 The Initial Solution obtained by VAM method

\begin{tabular}{|c|c|c|c|c|c|c|}
\hline & $\mathrm{D}_{1}$ & $\mathrm{D}_{2}$ & $\mathrm{D}_{3}$ & $\mathrm{D}_{4}$ & $D_{5}$ & $\begin{array}{l}\text { Supply } \\
\text { Source }\end{array}$ \\
\hline $\mathrm{O}_{1}$ & 68 & 35 & \begin{tabular}{l|l}
4 & 18 \\
\end{tabular} & 74 & 15 & 18 \\
\hline $\mathrm{O}_{2}$ & 57 & 88 & 91 & \begin{tabular}{|l|l|}
3 & 3
\end{tabular} & \begin{tabular}{l|l}
88 & 14
\end{tabular} & 17 \\
\hline $\mathrm{O}_{3}$ & \begin{tabular}{l|l}
91 & 16 \\
\cline { 2 - 2 }
\end{tabular} & \begin{tabular}{l|l}
60 & 3
\end{tabular} & 75 & 45 & 60 & 19 \\
\hline $\mathrm{O}_{4}$ & 52 & 53 & \begin{tabular}{l|l}
24 & 2 \\
\cline { 2 - 2 }
\end{tabular} & \begin{tabular}{l|l|}
7 & 11 \\
\cline { 2 - 2 }
\end{tabular} & 82 & 13 \\
\hline $\mathrm{O}_{5}$ & 51 & \begin{tabular}{|l|l|}
18 & 15 \\
\cline { 2 - 2 }
\end{tabular} & 82 & 13 & 7 & 15 \\
\hline Required & 16 & 18 & 20 & 14 & 14 & $82 / 82$ \\
\hline
\end{tabular}

Table-3 Second Basic Feasible solution task of VAM

\begin{tabular}{|c|c|c|c|c|c|c|}
\hline & $D_{1}$ & $D_{2}$ & $D_{3}$ & $\mathrm{D}_{4}$ & $D_{5}$ & $\begin{array}{l}\text { Supply } \\
\text { Source }\end{array}$ \\
\hline $\mathrm{O}_{1}$ & 68 & 35 & \begin{tabular}{|l|l|}
4 & 18 \\
\cline { 2 - 2 }
\end{tabular} & 74 & 15 & 18 \\
\hline $\mathrm{O}_{2}$ & 57 & 88 & 91 & \begin{tabular}{|l|l|}
3 & 3 \\
\end{tabular} & \begin{tabular}{|l|l|}
8 & 14 \\
\end{tabular} & 17 \\
\hline $\mathrm{O}_{3}$ & \begin{tabular}{|l|l|}
91 & 5 \\
\cline { 2 - 2 }
\end{tabular} & \begin{tabular}{|l|l}
60 & 14 \\
\cline { 2 - 2 }
\end{tabular} & 75 & 45 & 60 & 19 \\
\hline $\mathrm{O}_{4}$ & \begin{tabular}{|l|l|}
52 & 11 \\
\cline { 2 - 2 }
\end{tabular} & 53 & \begin{tabular}{|l|l}
24 & 2 \\
\end{tabular} & 7 & 82 & 13 \\
\hline $\mathrm{O}_{5}$ & 51 & \begin{tabular}{|l|l|}
18 & 4 \\
\end{tabular} & 82 & \begin{tabular}{|l|l|l|}
13 & 11 \\
\cline { 2 - 2 }
\end{tabular} & 7 & 15 \\
\hline Required & 16 & 18 & 20 & 14 & 14 & \\
\hline
\end{tabular}

This problem is not feasible since number of allocations are 8 where as $m+n-1=5+5-1=9$. Then the given solution is made feasible by allocating $\square \square$ units to the least cost, vacant independent cell ( $\left(\mathrm{g}, \mathrm{D}_{5}\right)$ and then, optimality test is performed. The IBFS took mine Iteration to get the solution while the optimal solution was achieved using transportation simplex algorithm within three iterations and the final cost was found to be $\mathrm{Zmin}=2$,202. Solution of the proposed method (IVAM) for the same problem is illustrated in the following section.

A detailed literature review on the basic solution methods is not presented. All the optimal an initial basic feasible solution to obtain the optimal solution.

\subsection{Variants of Improved Vogel's Approximation Method (IVAM)}

Since the basic idea of TOM is extended along with the VAM procedure, ToM is first briefly discussed here. The ToM is an effective application of the "best cell method" along with the tie breaking features on the total opportunity (cost) matrix. The TOC matrix is obtained by adding the row opportunity cost matrix (row opportunity cost matrix for each row, the smallest cost of that row is subtracted from each element of the same row) and the "Column opportunity cost matrix" (Column opportunity cost matrix for each column of the original transportation cost matrix the smallest cost of that column is subtracted from each lelement of the same column) [5]. Proposed algorithm is applied to the TOC matrix that considers highest three penalty cost and calculated alternative cost in the VAM procedure. It than selects the minimum among them. Detailed processes are given below:

Step: 1. Balance the given transportation problem if either (total supply $>$ total demand) or (total $<$ total demand).

Step: 2. Obtain ToC matrix 
Step: 3. Determine the penalty cost for each row and column by subtracting the lowest cell cost in the row or column from the next lowest cell in the same row or column.

Step: 4. Select the rows or columns with the highest three penalty cost (breaking ties arbitrarily or crossing the lowest - cost cell)

Step: 5. Compute three transportation cost for selected three rows or columns in Step 4 by allocating as much as possible to the feasible cell with the lowest transportation cost.

Step: 6. Select minimum transportation cost of three allocations instep 5 (breaking this arbitrarily or choosing the lowest-cost cell)

Step: 7. Repeat steps 3-6 until all requirements have been meet.

Step: 8. Compute total transportation cost for the feasible allocation using original balanced - transportation Remarks:cost matrix.

The Algorithm will be improved if we add the following two additional steps for breaking ties.

1. If there is a tie in penalty or minimum transportation cost, choose the largest penalty for allocation.

2. If there is a tie in penalty and minimum transportation cost, then calculate their corresponding row opportunity cost value/column opportunity cost value, and select the one with maximum.

Table -4 Initial solution table by IVAM

\begin{tabular}{|c|c|c|c|c|c|c|}
\hline & $D_{1}$ & $D_{2}$ & $D_{3}$ & $\mathrm{D}_{4}$ & $D_{5}$ & $\begin{array}{l}\text { Supply } \\
\text { Source }\end{array}$ \\
\hline $\mathrm{O}_{1}$ & 68 & 35 & \begin{tabular}{l|l}
4 & 18 \\
\end{tabular} & 74 & 15 & 18 \\
\hline $\mathrm{O}_{2}$ & \begin{tabular}{|l|l|}
57 & 3 \\
\end{tabular} & 88 & 91 & 3 & \begin{tabular}{l|l}
8 & 14 \\
\end{tabular} & 17 \\
\hline $\mathrm{O}_{3}$ & \begin{tabular}{|l|l|}
91 & 2 \\
\end{tabular} & \begin{tabular}{l|l}
60 & 13 \\
\end{tabular} & 75 & \begin{tabular}{l|l}
45 & 4
\end{tabular} & 60 & 19 \\
\hline $\mathrm{O}_{4}$ & \begin{tabular}{l|l|}
52 & 11 \\
\end{tabular} & 53 & $24 \underline{2}$ & 7 & 82 & 13 \\
\hline $\mathrm{O}_{5}$ & 51 & \begin{tabular}{|l|l|}
18 & 5 \\
\cline { 2 - 2 }
\end{tabular} & 24 & \begin{tabular}{l|l}
7 & 10 \\
\cline { 2 - 2 }
\end{tabular} & 82 & 15 \\
\hline Required & 16 & 18 & 20 & 14 & 14 & $82 / 82$ \\
\hline
\end{tabular}

For the transportation problem given in table -1 the initial solution of VAM requires five additional stages to reach to the optional solution. The problem was resolved using IVAM and IBFS for this problem is given in table-4 initial solution of IVAM is the optimal solution of the given example problem without additional iterations, initial cost from the table 4 is 2,247 and also is the optimal value of the considered problem.

\subsection{Computational experiments:-}

For evaluating the performance of the VAM and its variants and TOM, computational experiments were carried out. The experiments and the analysis of the experimental data are presented in this section. The main goal of the experiment was to evaluate the quality of the solution obtained by VAM and its variants and TOM by comparing them with optimal solution also, the iteration numbers to the optimal solution and computation times of VAM and improved version of IVAM

SIMULATION EXPERIMENTS:-

For evaluating the performance of the VAM and its variant IVAM, simulation experiments were carried out on a $2.13 \mathrm{GHz}$ Intel Core 2 Duo machine with 4096 MB RAM.

\subsection{Measure of effectiveness:-}

The performances of VAM and IVAM are compared using the following measures: The transportation problems were randomly generated with twelve different sizes (row x column) : 5x5, 10x10, 10x20, 10x30, $10 \times 40,20 \times 20,10 \times 60,30 \times 30,10 \times 100,40 \times 40,50 \times 50$, and 100x100, respectively. The experimental design was implemented using ANSI C.

a. Average Iteration (AI): Mean of iteration numbers to obtain optimal solutions using the initial solutions of VAM and IVAM over various sized problem instances.

b. Number of best solutions (NBS): A frequency which indicates the number of instances VAM and IVAM yielded optimal solution with lower iteration over the total of problem instances. NBS does not contain case of equal iteration between VAM and IVAM.

c. Computation Time: The CPU time is represented by three variables: $T_{a}, T_{b}$ and $T_{c} . T_{a}$ is the time to reach initial solution. $T_{b}$ is the time to reach optimal solution from initial solution and $T_{c}$ is the total time from the beginning that is sum of $T_{a}$ and $T_{b}$ 


\subsection{Comparison of VAM and IVAM:-}

The experiments and the analysis of the experimental data are presented in the section. For each problem instance, the heuristic solution were obtained using VAM and IVAM. The performance of the VAM and IVAM in comparison with the optimal solution is presented below. AI and other statistics for the iteration numbers of VAM and IVAM over various sized problems are given in Table 5.

Table 5. Statistical indicators for the iteration numbers of VAM and IVAM

\begin{tabular}{|c|c|c|c|c|c|c|c|c|}
\hline $\begin{array}{c}\text { Problem } \\
\text { Size }\end{array}$ & \multicolumn{2}{|c|}{ AI } & \multicolumn{2}{c|}{ Standard Error } & \multicolumn{2}{c|}{ Median } & \multicolumn{2}{c|}{ Range } \\
\hline & VAM & IVAM & VAM & IVAM & VAM & IVAM & VAM & IVAM \\
\hline 5 X5 & 2.198 & 2.676 & 0.034 & 0.35 & 2 & 3 & 5 & 5 \\
\hline $10 \times 10$ & 6.155 & 6.359 & 0.069 & 0.066 & 6 & 6 & 14 & 14 \\
\hline $10 \times 20$ & 10.063 & 9.913 & 0.094 & 0.092 & 10 & 10 & 18 & 18 \\
\hline $10 \times 30$ & 13.264 & 12.951 & 0.120 & 0.116 & 13 & 13 & 24 & 27 \\
\hline $10 \times 40$ & 17.761 & 17.495 & 0.141 & 0.144 & 17 & 17 & 28 & 30 \\
\hline $20 \times 20$ & 17.007 & 16.337 & 0.146 & 0.135 & 17 & 16 & 31 & 31 \\
\hline $10 \times 60$ & 22.869 & 22.118 & 0.173 & 0.166 & 23 & 22 & 36 & 32 \\
\hline $30 \times 30$ & 29.912 & 28.363 & 0.210 & 0.200 & 30 & 28 & 56 & 39 \\
\hline $10 \times 100$ & 32.561 & 31.139 & 0.221 & 0.220 & 32 & 31 & 40 & 43 \\
\hline $40 \times 40$ & 44.801 & 42.603 & 0.291 & 0.269 & 44 & 42 & 55 & 59 \\
\hline $50 X 50$ & 60.505 & 57.651 & 0.316 & 0.328 & 60 & 57 & 74 & 60 \\
\hline $100 \times 100$ & 158.890 & 149.53 & 0.657 & 0.610 & 158 & 149 & 137 & 112 \\
\hline
\end{tabular}

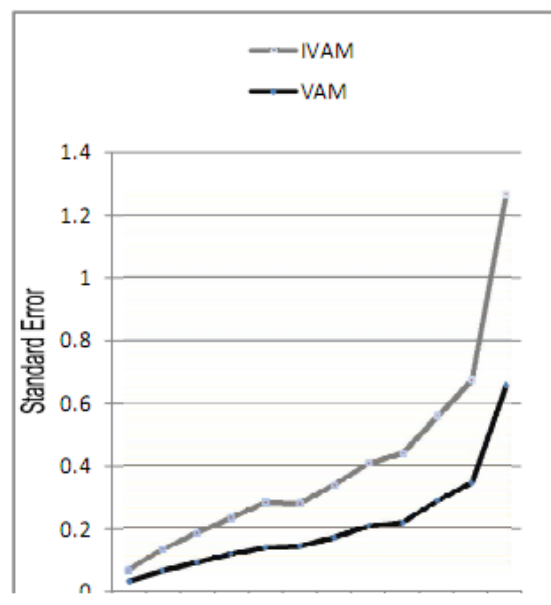

Fig 3- Graphical Representation of

Standard Errora

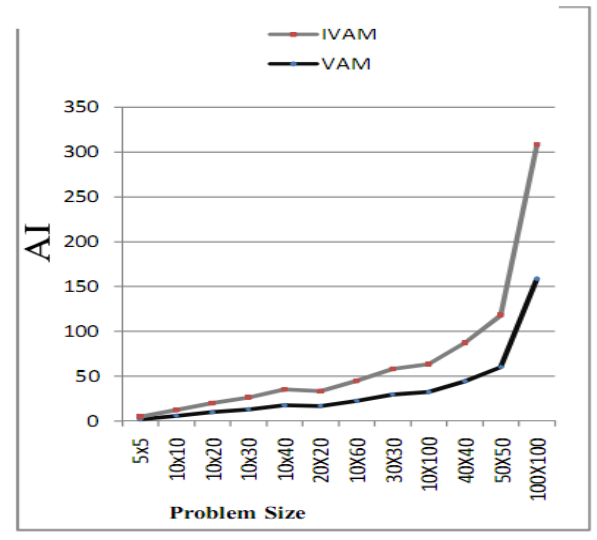

Fig 5- Graphical Representation of Average Iteration

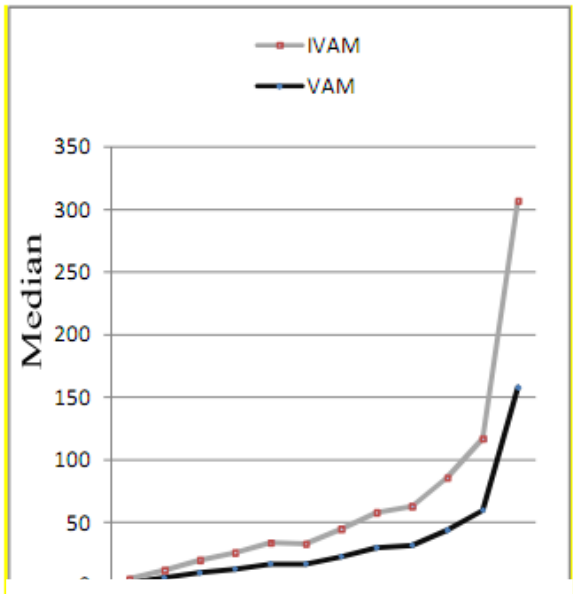

Fig 4- Graphical Representation of Median

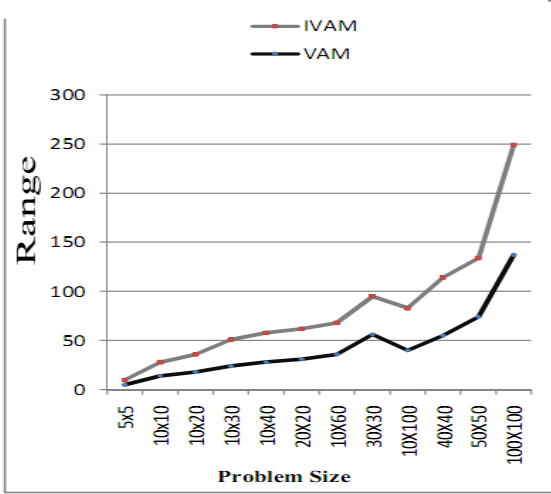

Fig. 6- Graphical Representation of Range 
Both parametric and nonparametric statistical tests were performed using MINITAB-15 Statistical Package for comparing iteration numbers of VAM and IVAM. Firstly, Students T-test was used for testing the mean of differences of the iteration numbers between VAM and IVAM based on 1000 samples. Secondly Wilcoxon test was used for testing the median of differences of the iteration numbers between VAM and IVAM on the same samples. Table 6 gives a summary of the results of two-sided statistical tests.

Table6. Statistical tests for difference of iteration numbers between VAM and IVAM

\begin{tabular}{|l|c|c|c|c|c|c|c|}
\hline \multirow{2}{*}{$\begin{array}{c}\text { Problem } \\
\text { size }\end{array}$} & $\begin{array}{c}\text { Mean } \\
\text { Standared } \\
\text { Error }\end{array}$ & $\begin{array}{c}\text { Confidence } \\
\text { Interval\%95 }\end{array}$ & $\mathbf{T}$ & $\mathbf{P}$ & $\begin{array}{c}\text { Estimated } \\
\text { Median }\end{array}$ & $\begin{array}{c}\text { Wilcoxon } \\
\text { Statistic }\end{array}$ & $\mathbf{P}$ \\
\hline $5 \times 5$ & $-0.478 \pm 0.039$ & $-0.555 ;-0.401$ & -12.190 & 0.000 & -0.500 & 52276.00 & 0.000 \\
\hline $10 \times 10$ & $-0.20 \pm .074$ & $-0.348 ;-0.060$ & -2.770 & 0.006 & 0.000 & 148409.00 & 0.008 \\
\hline $10 \times 20$ & $0.150 \pm 0.12$ & $-0051 ; 0.351$ & 1.470 & 0.143 & 0.000 & 20381.00 & 0.103 \\
\hline $10 \times 30$ & $0.313 \pm 0.125$ & $0.068 ; 0.558$ & 2.510 & 0.012 & 0.500 & 218149.500 & 0.022 \\
\hline $10 \times 40$ & $0.226 \pm 0.159$ & $-0.047 ; 0.579$ & 1.670 & 0.096 & 0.500 & 229297.500 & 0.104 \\
\hline $20 \times 20$ & $0.670 \pm 0.157$ & $0.362 ; 0.978$ & 4.270 & 0.000 & 0.500 & 245585.500 & 0.000 \\
\hline $10 \times 60$ & $0.751 \pm 0.191$ & $0.376 ; 1.126$ & 3.930 & 0.000 & 1.00 & 248683.500 & 0.000 \\
\hline $30 \times 30$ & $1.549 \pm 0.229$ & $1.099 ; 1.999$ & 6.760 & 0.000 & 1.500 & 281480.500 & 0.000 \\
\hline $10 \times 100$ & $1.422 \pm 0.249$ & $0.934 ; 1.910$ & 5.720 & 0.000 & 1.500 & 276839.00 & 0.000 \\
\hline $40 \times 40$ & $2.198 \pm 0.313$ & $1.584 ; 2.812$ & 7.030 & 0.000 & 2.500 & 290830.500 & 0.000 \\
\hline $50 \times 50$ & $2.85 \pm 0.375$ & $2.118 ; 3.590$ & 7.600 & 0.000 & 3.000 & 293811.500 & 0.000 \\
\hline $100 \times 100$ & $9.360 \pm 0.725$ & $7.937 ; 10783$ & 12.900 & 0.000 & 9.000 & 349416.00 & 0.000 \\
\hline
\end{tabular}

It is seen from Table 6 that, VAM has better results at the 0.01 significance level for the cases $5 \times 5$ and $10 \times 10$ problem sizes regarding both mean and median test. There is no difference at the 0.05 significance level between VAM and IVAM in the cases 10x20 and 10x30 problem sizes, regarding both mean and median test. On the other hand, in the rest of all the cases 10x10, 20x20, 10x60, 30x30, 10x100, 40x40, 50x50, and 100x100, both Student's t-test and Wilcoxon test show the same result the method of IVAM is statistically significantly different from the method of VAM.

d. Performance Measure- NBS: VAM and IVAM yield optimal solutions with different iteration numbers for different sized 1000 problem instances. These values are given in Table 7. From Table 7, it is clear that the NBS of VAM and IVAM significantly vary for different sized problems. Graphical representation of these values is shown in Figure.

Table 7. Number of best solutions

\begin{tabular}{|c|c|c|}
\hline Matrix size & \multicolumn{2}{|c|}{ NBS } \\
\hline Mxn & VAM & IVAM \\
\hline $5 \times 5$ & 460 & 183 \\
\hline $10 \times 10$ & 450 & 365 \\
\hline $10 \times 20$ & 420 & 455 \\
\hline $10 \times 30$ & 413 & 482 \\
\hline $10 \times 40$ & 430 & 499 \\
\hline $20 \times 20$ & 414 & 511 \\
\hline
\end{tabular}




\begin{tabular}{|c|c|c|}
\hline $10 \times 60$ & 406 & 523 \\
\hline $30 \times 30$ & 395 & 557 \\
\hline $10 \times 100$ & 394 & 564 \\
\hline $40 \times 40$ & 382 & 578 \\
\hline $50 \times 50$ & 395 & 587 \\
\hline $100 \times 100$ & 321 & 664 \\
\hline
\end{tabular}

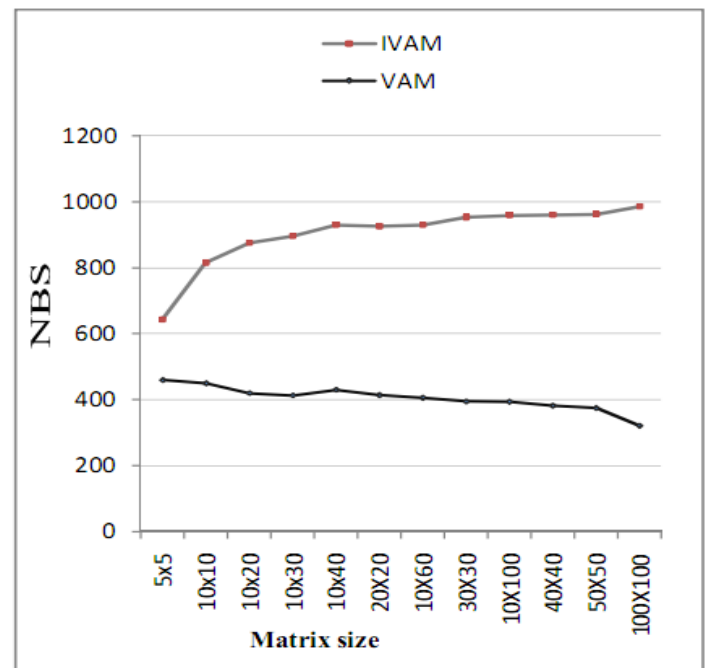

Fig. 7 Graphical Representation of Number of best solution

NBS does not include case of equal iteration between VAM and IVAM.

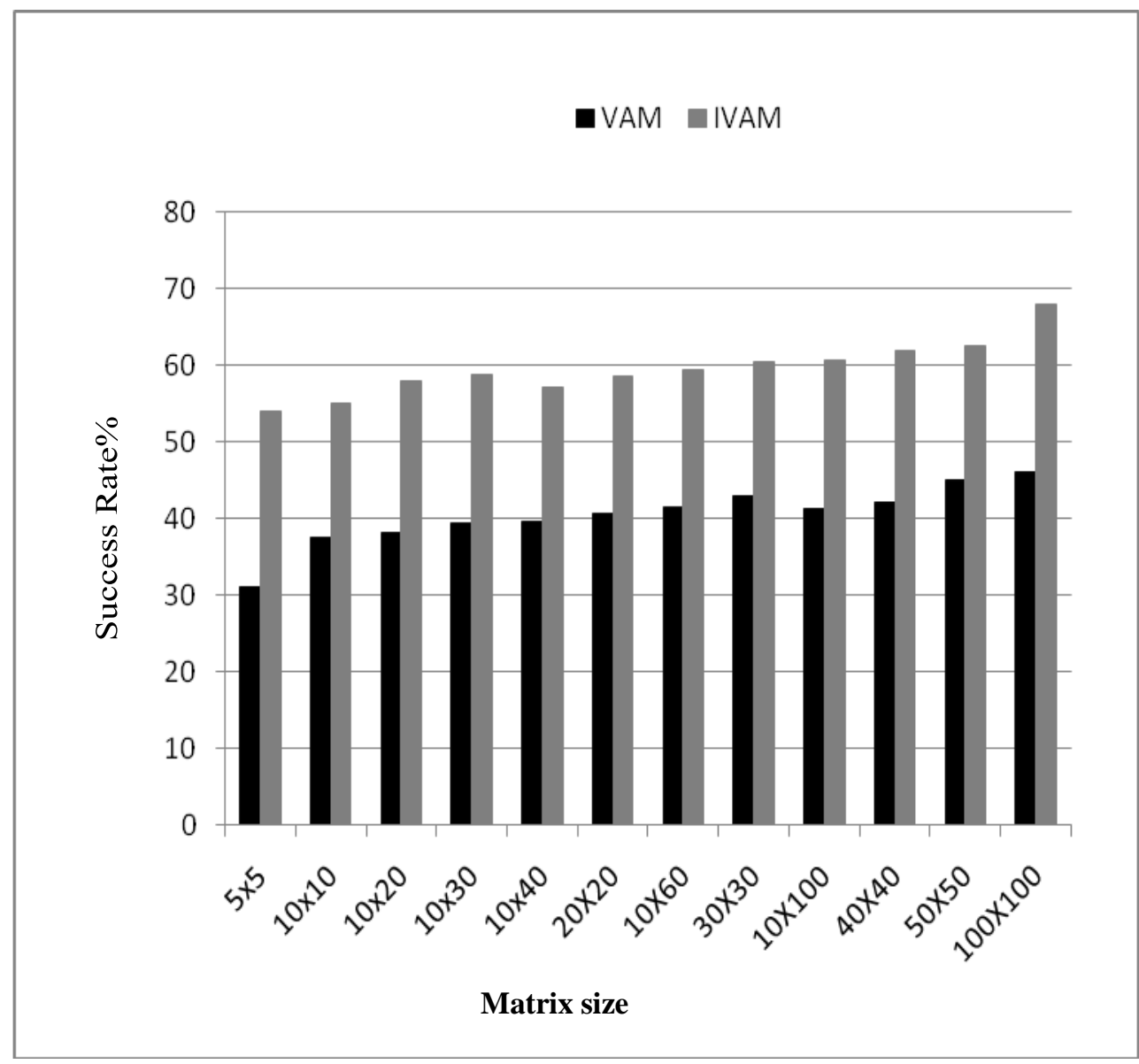

Fig. 8 Graphical Representation of Success rate 
Performance measure-CPU time: $\mathrm{T}_{\mathrm{a}}, \mathrm{T}_{\mathrm{b}}$ and $\mathrm{T}_{\mathrm{c}}$ times for VAM and IVAM over various sized problem instances are given in Table 8. For each different sized problem; mean, standard error, coefficient of variation and range of times are calculated based on 1000 samples.

Table $8 T_{a}, T_{b}$ and $T_{c}$ times for VAM and IVAM

\begin{tabular}{|c|c|c|c|c|c|c|c|}
\hline \multirow[t]{2}{*}{$\begin{array}{l}\text { Problem } \\
\text { Size }\end{array}$} & \multirow[t]{2}{*}{ Time } & \multicolumn{2}{|c|}{ Mean \pm Standard Error } & \multicolumn{2}{|c|}{$\begin{array}{c}\text { Coefficient of } \\
\text { Variation }\end{array}$} & \multicolumn{2}{|c|}{ Range } \\
\hline & & VAM & IVAM & VAM & IVAM & VAM & IVAM \\
\hline \multirow{3}{*}{$5 \times 5$} & $\mathrm{Ta}$ & $0.23 \pm 0.005$ & $1.335 \pm 0.003$ & 20.060 & 6.720 & 0.969 & 2.456 \\
\hline & $\mathrm{Tb}$ & $0.490 \pm 0.004$ & $0.532 \pm 0.023$ & 23.500 & 140.460 & 1.507 & 23.811 \\
\hline & Tc & $1.413 \pm 0.007$ & $1.867 \pm 0.024$ & 15.960 & 41.230 & 1.795 & 24.410 \\
\hline \multirow{3}{*}{$10 \times 10$} & $\mathrm{Ta}$ & $3.914 \pm 0.007$ & $4.395 \pm 0.255$ & 5.83 & 183.730 & 1.004 & 255.404 \\
\hline & $\mathrm{Tb}$ & $1.591 \pm 0.021$ & $1.599 \pm 0.012$ & 41.65 & 23.510 & 14.416 & 3.447 \\
\hline & Tc & $5.505 \pm 0.022$ & $5.995 \pm 0.256$ & 13.05 & 134.910 & 12.805 & 255.960 \\
\hline \multirow{3}{*}{$10 \times 20$} & $\mathrm{Ta}$ & $8.188 \pm 0.017$ & $9.115 \pm 0.059$ & 6.85 & 20.540 & 13.777 & 47.398 \\
\hline & $\mathrm{Tb}$ & $5.421 \pm 0.368$ & $4.853 \pm 0.054$ & 214.84 & 35.390 & 264.654 & 21.039 \\
\hline & Tc & $13.610 \pm 0.371$ & $13.969 \pm 0.090$ & 86.26 & 20.450 & 267.549 & 68.193 \\
\hline \multirow{3}{*}{$10 \times 30$} & $\mathrm{Ta}$ & $14.427 \pm 0.023$ & $14.700 \pm 0.046$ & 5.15 & 9.860 & 16.248 & 22.308 \\
\hline & $\mathrm{Tb}$ & $9.646 \pm 0.0778$ & $9.5091 \pm 0.082$ & 25.54 & 27.540 & 24.748 & 35.524 \\
\hline & Tc & $24.074 \pm 0.815$ & $24.209 \pm 0.094$ & 10.71 & 12.270 & 26.586 & 35.934 \\
\hline \multirow{3}{*}{$10 \times 40$} & $\mathrm{Ta}$ & $23.599 \pm 0.027$ & $23.908 \pm 0.046$ & 3.46 & 6.110 & 16.223 & 17.899 \\
\hline & $\mathrm{Tb}$ & $23.198 \pm 0.167$ & $22.872 \pm 0.175$ & 22.74 & 24.160 & 38.456 & 47.612 \\
\hline & Tc & $46.798 \pm 0.170$ & $46.780 \unlhd$ & 11.49 & 12.480 & 47.656 & 48.769 \\
\hline \multirow{3}{*}{$20 \times 20$} & $\mathrm{Ta}$ & $20.604 \pm 0.618$ & $20.790 \pm 0.049$ & 2.34 & 7.460 & 12.533 & 24.137 \\
\hline & $\mathrm{Tb}$ & $20.393 \pm 0.194$ & $19.415 \pm 0.178$ & 30.10 & 28.920 & 42.207 & 50.310 \\
\hline & Tc & $41.997 \pm 0.196$ & $40.205 \pm 0.185$ & 15.11 & 14.540 & 44.453 & 50.462 \\
\hline \multirow{3}{*}{$10 \times 60$} & $\mathrm{Ta}$ & $37.521 \pm 0.272$ & $37.134 \pm 0.044$ & 2.290 & 3.750 & 20.643 & 15.402 \\
\hline & $\mathrm{Tb}$ & $50.587 \pm 0.361$ & $49.185 \pm 0.345$ & 22.570 & 22.210 & 74.967 & 66.375 \\
\hline & Tc & $88.108 \pm 0.363$ & $86.319 \pm 0.347$ & 13.020 & 12.730 & 75.154 & 66.355 \\
\hline \multirow{3}{*}{$30 \times 30$} & $\mathrm{Ta}$ & $64.438 \pm 0.098$ & $60.469 \pm 0.147$ & 4.830 & 7.690 & 51.111 & 73.508 \\
\hline & $\mathrm{Tb}$ & $126.01 \pm 0.900$ & $119.450 \pm$ & 22.830 & 21.860 & 271.920 & 165.460 \\
\hline & Tc & $190.45 \pm 0.917$ & $0 \pm 0.858$ & 15.230 & 26.080 & 275.680 & 224.840 \\
\hline \multirow{3}{*}{$10 \times 100$} & $\mathrm{Ta}$ & $82.871 \pm 0.122$ & $75.701 \pm 0.144$ & 4.670 & 6.000 & 72.1770 & 49.895 \\
\hline & $\mathrm{Tb}$ & $182.6301 \pm 230$ & $175.180 \pm 1.720$ & 21.290 & 22.080 & 275.440 & 243.270 \\
\hline & Tc & $265.500 \pm 1.250$ & $250.880 \pm 1.250$ & 14.870 & 15.690 & 278.990 & 256.180 \\
\hline \multirow{3}{*}{$40 \times 40$} & $\mathrm{Ta}$ & $156.980 \pm 0.138$ & $143.22 \pm 0.341$ & 2.780 & 7.52 & 70.390 & 273.43 \\
\hline & $\mathrm{Tb}$ & $552.460 \pm 3.560$ & $526.340 \pm 3.290$ & 20.400 & 19.76 & 678.010 & 717.07 \\
\hline & Tc & $709.403 \pm 3.570$ & $669.550 \pm 3.310$ & 15.930 & 15.63 & 688.250 & 732.43 \\
\hline \multirow{3}{*}{$50 \times 50$} & $\mathrm{Ta}$ & $321.800 \pm 0.040$ & $276.580 \pm 0.075$ & 0.390 & 0.860 & 20.630 & 41.410 \\
\hline & $\mathrm{Tb}$ & $1662.900 \pm 9.440$ & $1585.700 \pm 8.960$ & 17.950 & 17.870 & 2029.700 & 1622.700 \\
\hline & Tc & $1984.700 \pm 9.440$ & $1862.300 \pm 8.960$ & 15.094 & 15.220 & 2029.300 & 1620.800 \\
\hline \multirow{3}{*}{$100 \times 100$} & $\mathrm{Ta}$ & $4737.7 \pm 1.440$ & $2625.400 \pm 0.646$ & 0.950 & 0.780 & 799.4 & 278.400 \\
\hline & $\mathrm{Tb}$ & $5181 \pm 214.000$ & $48760 \pm 199$ & 13.060 & 12.900 & 44226 & 37299 \\
\hline & Tc & $56549 \pm 214.000$ & $51386 \pm 199$ & 11.960 & 12.240 & 44226 & 37332 \\
\hline
\end{tabular}




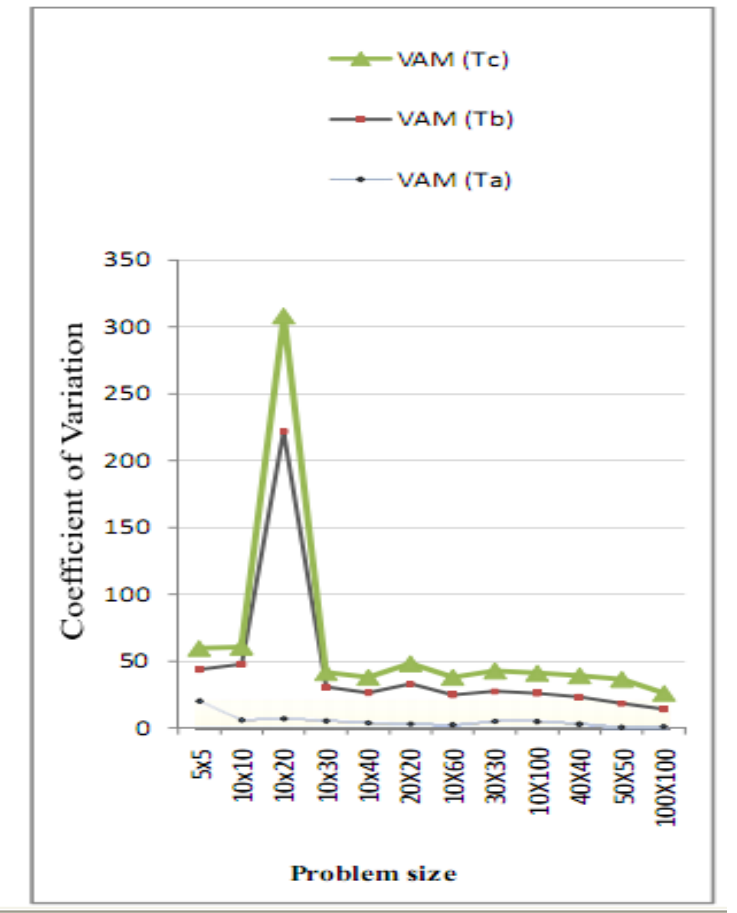

Fig. 9 Graphical Representation of Coefficient of Variation of VAM in $\left(\mathbf{T}_{\mathbf{a}}, \mathbf{T}_{\mathrm{b}}, \mathbf{T}_{\mathrm{c}},\right)$ Time

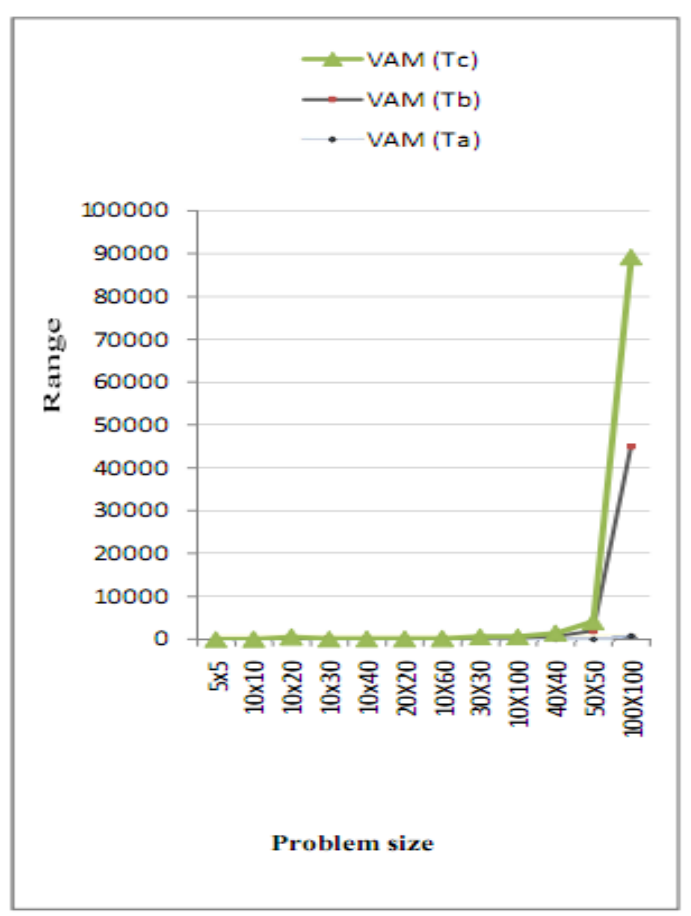

Fig 11- Graphical Representation of VAM in $\left(\mathrm{T}_{\mathrm{a}}, \mathrm{T}_{\mathrm{b}} \mathrm{T}_{\mathrm{c}}\right)$

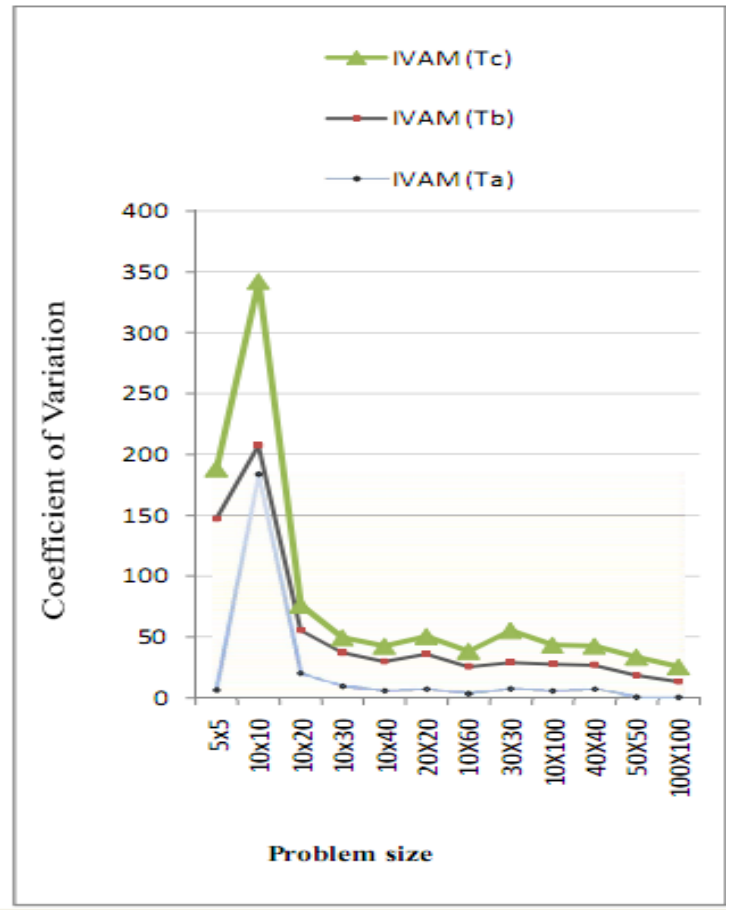

Fig. 10 Graphical Representation of Coefficient of Variation of IVAM in $\left(T_{a}, T_{b}, T_{c}\right)$ Time

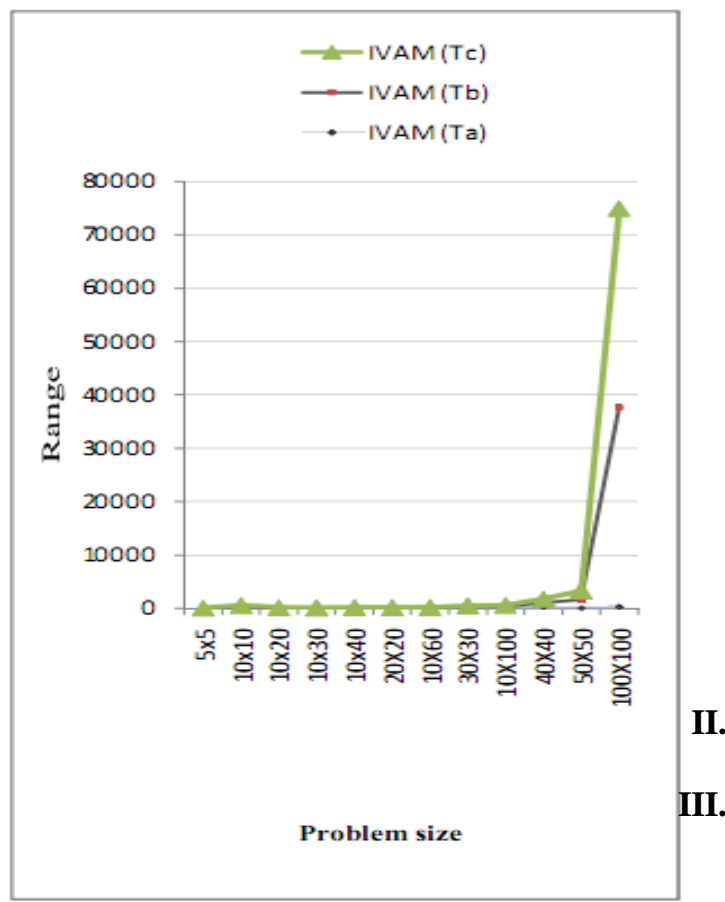

Fig 12- Graphical Representation of IVAM in $\left(\mathrm{T}_{\mathrm{a}}, \mathrm{T}_{\mathrm{b}} \mathrm{T}_{\mathrm{c}}\right)$ 


\section{IV.}

\section{CONCLUSION}

Vogel's approximation method (Penalty or regret method) is a heuristics method and is preferred always because it gives an initial solution which is wearer to an optimal solution or is the optimal solution itself. VAM was improved by using total opportunity cost and regarding alternative allocation costs. In analysis we found that the quality of solution in Basic version of VAM coupled with TOC yields a very efficient initial solution. Simulation experiments showed that VAM gets efficient initial solution for small sized transportation problems but it is insufficient for large sized transportation problem. IVAM conspicuously obtains more efficient initial solutions for large scale transportation times and computational difficulty for the optimal solution. A comparative study with the help of the graph is shown through various charts.

\section{REFERENCES}

[1] H. Wagner, Principles of Operations Research, New Jersey: PrenticeOHall, Englewood Cliffs, 1969.

[2] H.A. Taha, Operations Research: An Introduction, New York, : Macmillan Publishing Company, 1987.

[3] G.B. Dantzi, Linear Programming and Extensions, New, Jersey: Princeton University Press, 1963.

[4] J.E. Reeb and S. Leavengood, Transportation problem: A Special case for linear programming, Oregon: Oregon State University Extension Service Publications EM 8779, 2002.

[5] D.G. Shimshank, J.A. Kaslik and T.D. Barclay, A modification of Vogel's approximation method through the use of heuristics, infor 19, 259-263, 1981,

[6] S.K. Goyal, Improving VAM for unbalanced transportation problems, Journal of Operational Research Society 35 (12), 1113-1114, 1984.

[7] G.S. Ramakrishnan, An improvement to Goyal's modified VAM for the unbalanced transportation problem, Journal of Operational Research Society 39(6), 609-610-, 1988.

[8] O. Kirca and A. Satir, A heuristic for obtaining an initial solution for the transportation problem, Journal of Operational Research Society 41(9), 865-871, 1990.

[9] N. Balakrishnan, Modified Vogel's Approximation method for unbalanced transportation problem. Applied Mathematics Letters 3 (2), 9-11, 1990

[10] R.R.K. Sharma and S. Prasad, Obtaining a good primal solution to the incapacitated transportation problem, European Journal of Operational Research 144, 560-564, 2003

[11] M. Mathirajan and B. Meenakshi, Experimental Analysis of some Variants of Vogel's Approximation Method, AsiaPacific Journal of Operational Research 21(4), 447-462, 2004.

[12] H.H. Shore, The Transportation Problem and the Vogel Approximation Method, Decision Sciences 1 (3-4), 441-457, 1970. 